\title{
Influence d'une co-construction de rationnement amélioré sur les performances d'embouche ovine paysanne dans la commune rurale de Korsimoro au Burkina Faso
}

\author{
B. Isidore GNANDA ${ }^{1 *}$, Man NIGNAN $^{1}$, Souleymane OUEDRAOGO ${ }^{1}$, \\ Aïssata WEREME N'DIAYE ${ }^{1}$, Oumar TRAORE ${ }^{2}$ et Boukaré SINON ${ }^{1}$

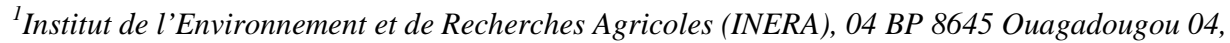 \\ Burkina Faso. \\ ${ }^{2}$ Unité de Formation et de Recherche en Science de la Vie et de la Terre (UFR/SVT), Département de \\ Biologie et Physiologie Végétale (BV/PV), 03 B.P. 7021 Ouagadougou 03, Burkina Faso. \\ *Auteur correspondant; E-mail: gnandaisid@yahoo.fr; Tél. : (+226) 50319208 , (+226) 70281118.
}

\section{RESUME}

L'étude vise l'amélioration des performances d'embouche ovine dans la commune rurale de Korsimoro. Quatre vingt douze (92) béliers de 22 femmes et de 24 hommes de cette commune ont été étudiés. L'équipe de recherche a défini quatre (4) formules de ration sur la base d'un seul profil nutritionnel apportant $98 \mathrm{~g}$ MAD (matières azotées digestibles) et $0,80 \mathrm{UF} / \mathrm{kg}$ MS. Les producteurs ont analysé ces formules et ont porté leur choix sur celle composée de $15 \%$ de tourteau de coton, $6 \%$ de son de blé, $31 \%$ de graines de coton et $48 \%$ de gousses de Piliostigma reticulatum. Cette ration a été distribuée à deux lots d'animaux en comparaison avec les pratiques locales : lot1=aliment composé introduit+fourrages locaux et lot2=aliment composé introduit+fourrages locaux+bloc multinutritionnel. Les GMQ des animaux du lot 2 ont été significativement $(\mathrm{P}<0,05)$ plus élevés que ceux des animaux du lot 1 . La croissance des animaux soumis sous rationnement amélioré $(75 \mathrm{~g} / \mathrm{j})$ a été très significativement $(\mathrm{P}<0,01)$ plus élevée que celle enregistrée des pratiques paysannes (environ $46 \mathrm{~g} / \mathrm{j}$ ). Une marge bénéficiaire moyenne de $9173 \mathrm{~F}$ CFA a été dégagée par ovin embouché. Les résultats de l'étude suggèrent la nécessité d'améliorer le dispositif organisationnel des emboucheurs.

(C) 2015 International Formulae Group. All rights reserved.

Mots clés: Embouche ovine, gain moyen quotidien, Burkina Faso, bloc multinutritionnel, plateforme d'innovation.

\section{INTRODUCTION}

Un des défis majeurs que la plupart des pays de l'Afrique de l'Ouest et du Centre doit relever, est de parvenir à assurer un niveau de sécurité alimentaire adéquat à leur population de plus en plus croissante. Le Burkina Faso, en tant que pays d'élevage, peut compter sur ce secteur pour relever en partie ce défi. En effet, il est le deuxième sous-secteur productif du secteur agricole et touche directement la plus grande proportion de pauvres $(43,9 \%$ d'incidence de pauvreté en 2009) selon la SCADD (2007). Il est pratiqué par environ $82 \%$ des ménages agricoles du 
pays (MAHRH, 2007). Dans ce sous-secteur, l'élevage ovin et en particulier, la pratique de l'embouche ovine est d'une importance capitale dans la vie des populations rurales burkinabé (Zoundi et al., 2003). Cependant, l'essor de cette activité reste toujours limité par un certain nombre d'entraves. D'abord, il y a le faible niveau de maîtrise technique des emboucheurs ruraux (Nianogo et al., 1997 ; Somda, 2001). En plus, Il y a les contraintes liées à l'inexistence des conditions favorables à l'application des innovations préconisées par la recherche telles que la faible accessibilité et la qualité limitée d'un bon nombre d'intrants zootechniques hors-fermes (Somda, 2001; Gnanda et al., 2008), l'insuffisance d'instruments ou de moyens adaptés au dosage et à la distribution des quantités recommandées pour ces intrants aux animaux (Lodoun, 2012). A ces contraintes, s'ajoutent celles liées au manque d'opportunités intéressantes de marchés capables d'inciter les éleveurs à s'investir résolument dans les modèles d'alimentation améliorés élaborés par la recherche (Yacouba et al., 2004).

La présente étude a eu pour objet d'améliorer les performances d'embouche ovine paysanne dans la commune rurale de Korsimoro à travers une démarche de coformulation et d'application participative d'un rationnement amélioré des animaux. Cette recherche a été réalisée dans le cadre de la mise en œuvre d'un projet sous financement compétitif du Conseil Ouest et Centre Africain pour la Recherche et le Développement Agricole/ West and Central African Council for Agricultural Research and Development (CORAF / WECARD). L'hypothèse de base de recherche qui a guidé la mise en œuvre du projet a été que l'intégration des cultures et de l'élevage à l'échelle des exploitations et du paysage, peut contribuer à accroître durablement la productivité agricole des pays sahéliens.

\section{MATERIEL ET METHODES \\ Traits caractéristiques des ovins embouchés}

Un échantillon de 92 béliers a fait l'objet d'étude et constitué sans imposition préalable de critère particulier de choix des animaux en termes d'âge, de poids ou de race. L'état de lieu qui a été fait après constitution de cet échantillon d'animaux, a montré qu'ils étaient des mâles entiers, âgés de 8 à 24 mois et de race Djallonké variété Mossi pour la plupart (90\% d'entre eux). Les 10\% restant des sujets, étaient de sang métissé (croisement entre mouton Djallonké variété Mossi et mouton sahélien). La plus grande proportion de ces ovins (environ 70\% des sujets utilisés) a été achetée et le reste a été prélevé des troupeaux familiaux. Les ateliers d'embouche ovine de deux (2) têtes d'ovins étaient les plus nombreux $(73 \%$ des ateliers d'embouche ovine).

\section{Identification participative du besoin d'appui en technologie d'embouche ovine}

L'approche d'intervention du projet a été basée sur la démarche de recherche agricole intégrée pour le développement (RAIPD). Avant la mise en œuvre du projet sur le terrain, un atelier de concertation nationale a été organisé par l'équipe de recherche et a permis à tous les acteurs directs et indirects dudit projet de partager l'information sur sa démarche et sur les activités à mener. Les échanges qui ont nourri cette rencontre nationale ont permis l'identification de trois (3) chaînes de valeur phares à performer dans la commune de Korsimoro dont celle du bétail/viande.

Les enquêtes diagnostiques qualitatives qui ont été réalisées par la suite dans cette commune à l'aide des outils MARP (Méthode active de recherche participative) ont permis de confirmer l'importance et la place de la chaîne de valeur bétail-viande dans 
l'intensification agricole des exploitations paysannes de la localité.

Identification et développement avec les acteurs (producteurs) de la formule de rationnement des ovins

Co-identification du rationnement à appliquer dans les ateliers d'embouche

A l'étape du choix de rationnement à appliquer, l'équipe de recherche a d'abord défini quatre (4) formules de ration sur la base d'un seul profil nutritionnel assurant un niveau d'apport de $98 \mathrm{~g}$ MAD et $0,80 \mathrm{UF} / \mathrm{kg}$ MS. Ces formules de ration ont été placées auprès des producteurs qui les ont analysées, puis ont opéré leur choix en tenant compte de l'accessibilité des matières premières (aliments) qui entrent dans leur composition. Le choix définitif des producteurs à l'issue de cette démarche de co-identification a porté sur la formule $\mathrm{D}$ qui se compose de $15 \%$ de tourteau de coton, $6 \%$ de son de maïs, $31 \%$ de graines de coton et $48 \%$ de gousses ou fruits de ligneux (Tableau 1).

\section{Choix des expérimentateurs et constitution de l'échantillon d'animaux utilisés}

Une fois que le choix de la formule de rationnement a été opéré, l'équipe de recherche a informé et sensibilisé les producteurs sur la base d'un cahier de charges pour le recrutement en leur sein, des candidats à la mise en œuvre de l'expérimentation. Les principaux termes qui ont constitué les closes de ce cahier de charges étaient les suivants :

- fournir les animaux d'embouche (un minimum requis de deux sujets) ;

- stocker suffisamment de fourrage pour mener à terme l'expérimentation ;

- s'engager à mesurer et distribuer quotidiennement les rations des animaux ;

- construire une fosse compostière pour la valorisation des déjections animales et des refus alimentaires collectés des ateliers d'embouche ;

- être disponibles et collaborer, de manière effective, pour la mise en œuvre de l'étude : être présents aux jours de suivi, aux séances de collecte de données, aux visites commentées, etc. ;

- assurer la sécurité des animaux et leur disponibilité pour les mesures à réaliser.

Au total, 46 exploitants (22 femmes et 24 hommes) ont pris part à l'étude avec leurs 92 ovins mâles qui ont été mis en embouche et suivis à terme. Ces animaux ont été préalablement soumis au protocole de prophylaxie sanitaire en vigueur dans la région. Les mesures de prophylaxie ont été assurées par le personnel du service d'encadrement en élevage du ministère des ressources animales résident dans la commune de Korsimoro.

Rationnement des animaux dans les ateliers d'embouche

La formule de ration qui a été appliquée (aliment composé D) a d'abord été fabriquée industriellement par un fabricant d'aliment privé sous forme de composé en poudrette avant d'être apportée aux paysans dans des sacs de $50 \mathrm{~kg}$. Au niveau des fermes d'embouche, les animaux ont été soumis à deux modalités d'alimentation durant 60 jours. La première modalité (lot 1) a concerné uniquement l'aliment composé apporté par la recherche (ration D) en complément aux fourrages locaux et la deuxième modalité (lot 2) a porté sur le même régime alimentaire (aliment D+fourrages locaux), tout en associant une complémentation d'appoint à l'aide de Bloc multinutritionnel (BMN) de fabrication artisanale. Ce BMN était composé d'urée $(10 \%)$, de tourteau de coton (35\%), de son de maïs $(7,5 \%)$, de gousses de Piliostigma reticulatum $(7,5 \%)$, de ciment (12\%), de kaolin $(5 \%)$, de coquilles d'huîtres $(11 \%)$ et de sel de cuisine (12\%). En résumé, le dispositif expérimental mis en place était le suivant :

- Lot 1= Aliment composé industriel D + fourrages locaux

- Lot 2= Aliment composé D + fourrages locaux+ BMN 
$\begin{array}{rrr}\text { Les } & \text { performances des } & \text { pratiques } \\ \text { paysannes } & \text { d'embouche } & \text { (conduite }\end{array}$ traditionnelle des acteurs) ont été appréciées durant 30 jours de suivi avant l'application du rationnement amélioré et ont servi de données témoins (données de référence) à l'innovation introduite.

Les quantités de l'aliment composé D distribuées aux animaux ont été déterminées sur la base de 2,7 kg MS/100 kg de leur poids vif (PV) et l'apport de la composante fourrage local a été fixé à $1,5 \mathrm{~kg}$ MS/100 kg du PV de chaque animal. Les BMN étaient mis à la consommation ad libitum aux animaux et les producteurs ont constitué les répétitions de chaque modalité d'alimentation testée.

Le rythme de distribution des aliments était en moyenne, deux (2) distributions par jour. L'aliment composé D de la recherche était distribué à l'aide de mesurettes faites d'instruments locaux (boîte vide de lait de 1 $\mathrm{kg}$, petite boîte vide de tomate de $400 \mathrm{~g}$ et petit bol en plastique). Ces mesurettes ont été calibrées à la quantité distribuée quotidiennement. Ce qui permettait aux emboucheurs de respecter les doses recommandées pour chaque animal.

\section{Caractérisation, suivi et collecte de données}

Tous les ateliers d'embouche des producteurs qui ont pris part à l'étude, ont fait l'objet de caractérisation et des suivis périodiques de 15 jours et ont permis de collecter les données relatives à l'alimentation des animaux et aux performances pondérales exprimées. En plus de ces suivis périodiques de 15 jours, un suivi alimentaire quotidien a été réalisé auprès d'un échantillon de 12 ateliers d'embouche ( 24 animaux). Ce suivi a permis de collecter les informations sur les différentes ressources alimentaires utilisées dans les unités d'embouche et les quantités distribuées aux animaux et les parts des refus alimentaires (données collectées à l'aide de pesons à ressort de $3 \mathrm{~kg}$ de portée et $50 \mathrm{~g}$ de précisons). Cela a permis d'apprécier l'ingestion volontaire des rations par les animaux et d'évaluer l'indice de consommation (IC).

Des prélèvements d'échantillons d'aliments ont été également réalisés à des fins d'analyses bromatologiques de laboratoire.

La pesée des animaux a été réalisée une fois tous les 15 jours à l'aide d'un peson à ressort de marque SALTER de $50 \mathrm{~kg}$ de portée et de $200 \mathrm{~g}$ de précision. Afin de faciliter le repérage des sujets et l'enregistrement des données, les animaux de l'étude ont été identifiés par numérotation à l'aide de plaquettes en contreplaqué.

\section{Analyses de laboratoire}

Les analyses de laboratoire ont porté sur la détermination de la matière sèche, de la matière minérale (MM), de la matière organique (MO) et les matières azotée totales (MAT). La matière sèche (MS) des échantillons a été déterminée après leur séchage dans une étuve à la température de $105{ }^{\circ} \mathrm{C}$ pendant 24 heures. Après l'étuvage, les échantillons ont été pesés et mis au four à moufle à $550{ }^{\circ} \mathrm{C}$ pendant 2 heures pour obtenir les cendres ou matières minérales (MM). Le dosage des matières azotées totales (MAT) a été réalisé selon la méthode KJELDAHL qui comporte une étape de minéralisation des échantillons à l'acide sulfurique concentré et une étape de distillation, suivie de titrage des distillats.

\section{Analyse économique}

Il s'est agi de déterminer la rentabilité économique des fermes suivies, en calculant quelques indicateurs clés. Ces indicateurs suivants sont calculés: marge brute, marge nette et productivité du capital

\section{Exploitation et analyse statistique des données}

Tous les paramètres collectés ont été saisis sur Excel pour en constituer une base de données. L'exploitation et l'analyse des données relatives aux caractéristiques des emboucheurs et de leurs ateliers d'embouche, 
ont été réalisées à l'aide du tableau croisé dynamique du logiciel Excel. Les données pondérales et d'alimentation ont fait l'objet d'analyse de variance (ANOVA) à partir du logiciel libre R (R Developement Core Team). $\mathrm{Au}$ regard de la variabilité des poids des animaux au départ, ils ont été utilisés comme co-variables dans l'analyse des performances pondérales enregistrées au cours de l'expérimentation.

\section{RESULTATS}

Composition et ingestion des ressources alimentaires utilisées dans l'alimentation des animaux

Le suivi des pratiques paysannes d'embouche a montré que les sous-produits agro-industriels (pellettes de tourteau coton) ont été utilisés dans des proportions de 2 à $5 \%$ des quantités des rations distribuées. La participation du son local de céréales à la composition de ces rations paysannes a été évaluée entre 6 et $8 \%$, celle des glumes de sorgho à environ 3\%. Les pailles de sorgho ont été les plus utilisées car ayant contribué à plus de $60 \%$ de la composition de ces rations paysannes. Les fanes de légumineuses ont contribué pour 13 à $21 \%$ à la composition de ces rations et les fruits de Faidherbia albida pour environ $2 \%$.

Globalement, les quantités des rations paysannes distribuées ont été évaluées entre 1,86 et $2,72 \mathrm{~kg} / \mathrm{animal} /$ jour, soit des valeurs d'offre de 8 à $11 \mathrm{~kg} \mathrm{MS} / 100 \mathrm{~kg}$ de poids vif.

Hormis les fanes de niébé et le son local de sorgho (Tableau 2), la plupart des aliments locaux utilisés dans l'alimentation des animaux d'embouche, avaient des valeurs en matière azotée totale (MAT) à la limite du taux minimum (6 à $8 \%$ ) nécessaire pour le bon fonctionnement de la microflore du rumen des ovins (Van Soest, 1994).

Les aliments locaux les plus utilisés en termes de quantité ou de fréquence dans le rationnement des animaux sont par ordre décroissant, les pailles de sorgho, les fanes et les cosses de niébé, les fanes d'arachide, les gousses de Faidherbia albida et le son de sorgho.
Ingestion alimentaire des rations distribuées

Durant l'application du rationnement amélioré de la recherche, les quantités brutes de fourrages distribuées aux animaux ont été évaluées en moyenne à $1,68 \pm 1,3 \mathrm{~kg}$, avec un niveau de consommation d'environ $49 \%$, soit une ingestion volontaire moyenne de $77 \mathrm{~g}$ MS/kg PM (poids métabolique). L'ingestion volontaire globale (aliment composé D + fourrages locaux) a été légèrement mais non significativement plus élevée au niveau du lot 1 (aliment D sans BMN) qu'au niveau du lot 2 (aliment D associée au BMN). En effet, la valeur enregistrée chez les animaux du lot 1 a été de $104 \pm 61$ g MS/kg PM contre $98 \pm 16$ g $\mathrm{MS} / \mathrm{kg}$ PM chez les ovins du lot 2. Par contre, en termes d'indice de consommation (IC), le rationnement du lot 2 a été plus performant, avec une valeur d'IC de 18,9 contre un IC de 24 pour le rationnement du lot 1 durant les 60 jours d'observation.

\section{Performances zootechniques des animaux et analyse financière des ateliers d'embouche}

Les résultats en termes d'évolution pondérale (Figure 1), montrent un accroissement significatif du poids des animaux des deux lots durant l'expérimentation. Les animaux du lot 1 ont vu leur poids accroître en valeur absolue de $5,4 \mathrm{~kg}$ contre $5,6 \mathrm{~kg}$ pour ceux du lot 2 . L'avance de croissance des animaux du lot 1 (GMQ) durant la phase d'alimentation sous rations paysannes, a subi en fin d'expérimentation sous le rationnement amélioré, une inversion significative de tendance à la faveur de la croissance des animaux du lot 2.

Durant la phase de l'application du rationnement amélioré, les GMQ exprimés par les animaux du lot 2 ont été significativement plus élevés que ceux enregistrés sur les animaux du lot 1 (Tableau 3).

Globalement, la croissance des animaux sous le rationnement amélioré introduit par la recherche a été très significativement $(\mathrm{P}<0,001)$ plus élevée que celle enregistrée sous les pratiques paysannes 
exclusives (Tableau 3). En effet, le GMQ des animaux durant la période 2 (période durant laquelle les animaux ont bénéficié du rationnement amélioré de la recherche) a été en moyenne de 75,54 \pm 39,11 g contre une valeur de 46,65 $\pm 34,53 \mathrm{~g}$ en moyenne pour la période 1 (période des 30 jours d'observations sous l'alimentation paysanne uniquement).

Les résultats de l'étude ont montré également que l'âge des animaux à l'entrée d'embouche a eu une influence, mais non significative sur les performances pondérales exprimées. Les jeunes béliers (animaux âgés de moins de 12 mois) ont eu en fin d'opération, des performances pondérales globalement plus intéressantes que les sujets de 12 à 18 mois d'âge et ceux de plus de 18 mois d'âge (Tableau 3).

Sous le rationnement amélioré de la recherche, les performances pondérales des animaux des femmes ont été plus légèrement intéressantes que celles des animaux des hommes. En effet, les GMQ des animaux des femmes sous ce régime ont été en moyenne de $78,47 \pm 36,16$ g contre en moyenne $75,39 \pm 32,23 \mathrm{~g}$ pour les animaux des hommes (Tableau 3).

L'analyse financière réalisée sur la base d'un compte d'exploitation d'un atelier d'embouche ovine de deux (2) d'ovins (taille moyenne des ateliers ayant fait l'objet de suivi), montre globalement que l'embouche ovine procure des marges bénéficiaires relativement intéressantes pour les emboucheurs de la commune rurale de Korsimoro (Tableau 4). Une marge bénéficiaire moyenne de 9173 F CFA par tête d'ovin embouché a été dégagée avec un ratio avantage/coût de 1,27 (pour 1 F CFA investi dans l'embouche, le promoteur a encaissé un surplus de 0,27 F CFA).

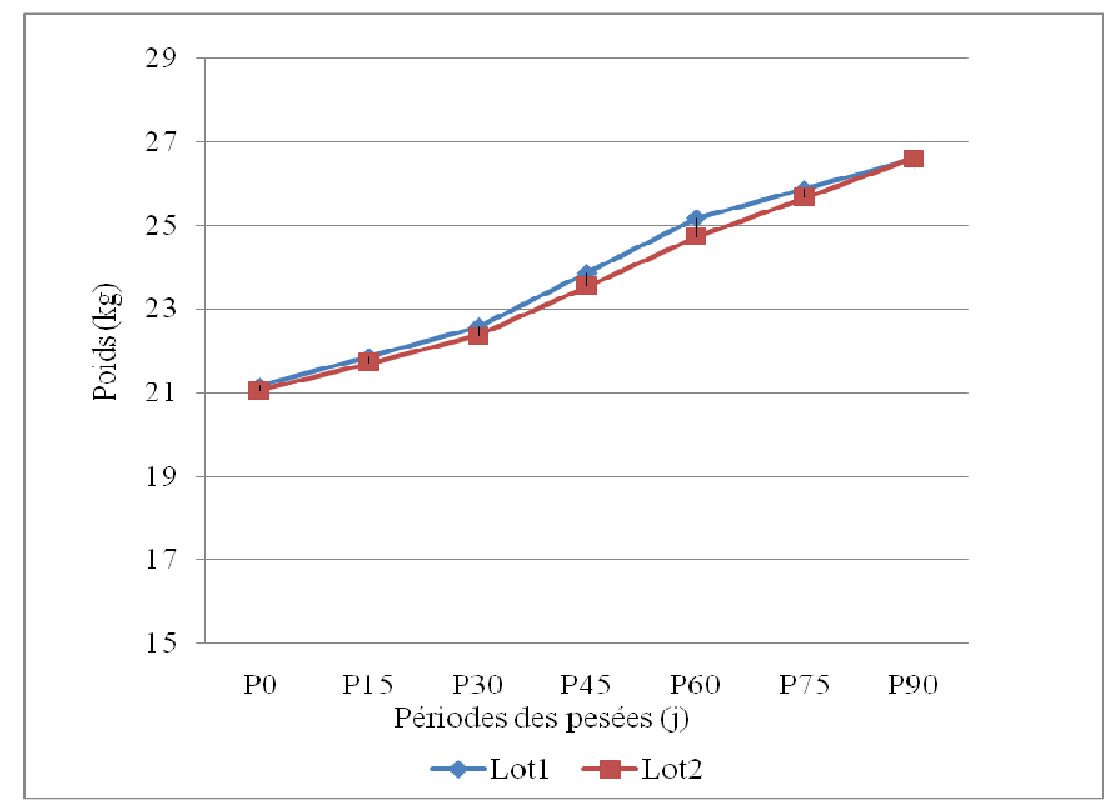

Figure 1: Evolution pondérale des ovins d'embouche. Lot 1 (Traitement1) = fourrage locaux + aliment composé alimentaire industriel (15\% de tourteau de coton, $6 \%$ de son maïs, $31 \%$ de graines de coton et $48 \%$ de gousses de Piliostigma reticulatum); Lot 2 (Traitement 2) = Traitement $1+$ Bloc multi nutritionnel $(10 \%$ d'urée, $35 \%$ de tourteau de coton, 7,5\% de son de maïs, 7,5\% de gousses de Piliostigma reticulatum, $12 \%$ de ciment, 5\% de kaolin, 11\% de coquilles d'huîtres et 12\% de sel de cuisine) ; P0-P30 : 30 jours d'embouche paysanne ; $\mathrm{P} 30-\mathrm{P} 90$ : 60 jours d'embouche à base de la ration proposée par la recherche. 
Tableau 1 : Les quatre (4) formules de ration soumises au choix des producteurs.

\begin{tabular}{lcccc}
\hline Matières premières & \multicolumn{4}{c}{ Formules alimentaires composées } \\
& \multicolumn{4}{c}{ (\%) } \\
\cline { 2 - 5 } & A & B & C & D \\
\hline Maïs grains & & 32 & & \\
Sorgho grains & & & 35 & \\
Tourteaux coton & 14 & 20 & 17 & 15 \\
Son de blé & 36 & 9 & 13 & \\
Son de maïs & & & & 6 \\
Graines de coton & & 39 & 35 & 31 \\
Gousses & 50 & & & 48 \\
TOTAL & 100 & 100 & 100 & 100 \\
\hline
\end{tabular}

Tableau 2 : Composition chimique des principaux aliments utilisés dans les ateliers d'embouche de Korsimoro.

\begin{tabular}{lcccc}
\hline Aliments & \%MS & \%MM & \%MO & \%MAT \\
\hline Paille de sorgho local & 93,39 & 7,94 & 92,05 & 3,70 \\
Foin de Pennisetum pedicellatum & 95,64 & 11,19 & 88,80 & 9,42 \\
Paille de riz & 96,38 & 25,52 & 74,47 & 3,13 \\
Andropogon gayanus (pailleux) & 96,47 & 7,38 & 92,62 & 2,1 \\
Son de riz & 93,56 & 14,68 & 85,13 & 7,69 \\
Fanes d'arachide & 93,70 & 18,82 & 81,17 & 8,38 \\
Fanes de niébé & 92,44 & 7,59 & 92,40 & 14,69 \\
Cosses de niébé & 93,04 & 10,23 & 89,76 & 6,28 \\
Tiges de mil & 94,31 & 8,12 & 91,87 & 4,9 \\
Sorgho fourrager & 95,16 & 9,05 & 90,94 & 6,62 \\
Glumes de sorgho & 93,78 & 4,28 & 95,71 & 8,72 \\
Rachis de maïs & 95,06 & 12,02 & 87,97 & 2.5 \\
Son local (sorgho) & 93,77 & 5,12 & 94,87 & 10,12 \\
Faidherbia albida & 95,53 & 5,76 & 94,24 & 5,06 \\
Piliostigma reticulatum & 94,73 & 5,31 & 94,69 & 6,61 \\
SPAI (pellettes tourteau de coton) & 95,77 & 6,21 & 93,78 & 29,72 \\
\hline
\end{tabular}

DPA/INERA, (2013), laboratoire de nutrition animale et de biologie animale du Département Productions Animales (DPA) à Kamboinsé, au Burkina Faso; $\mathrm{NB}: \mathrm{MS}=$ matière sèche; $\mathrm{MM}=$ matière minérale; $\mathrm{MO}=$ matière organique $; \mathrm{MAT}=$ matière azotée totale $; \mathrm{SPAI}=$ Sous-produits agro-industriel. 
Tableau 3 : Gain moyen quotidien (GMQ) des animaux par lots, par classes d'âge et par périodes (moyennes + écart types).

\begin{tabular}{|c|c|c|c|}
\hline Facteurs & GMQ P1 (g) & GMQ P2 (g) & GMQ P3 (g) \\
\hline \multicolumn{4}{|l|}{ Effet Type de rationnement : } \\
\hline Lot 1 (45 ovins)) & $52,23 \pm 36,71^{\mathrm{a}}$ & $71,82 \pm 34,16^{\mathrm{a}}$ & $62,57 \pm 30,13$ \\
\hline Lot 2 (47 ovins) & $41,13 \pm 25,22^{\mathrm{a}}$ & $78,71 \pm 39,77^{\mathrm{b}}$ & $61,21 \pm 26,83^{\circ}$ \\
\hline \multicolumn{4}{|c|}{ Effet âge des animaux en début essai : } \\
\hline Classe 1 (61 ovins) & $54,47 \pm 39,28^{\mathrm{a}}$ & $72,62 \pm 39,58^{\mathrm{a}}$ & $64,02 \pm 29,82^{2}$ \\
\hline Classe 2 (22 ovins) & $26,63 \pm 33,46^{\mathrm{a}}$ & $79,73 \pm 28,59^{a}$ & $56,41 \pm 24,05^{\circ}$ \\
\hline Classe 3 (9 ovins) & $31,59 \pm 39,61^{\mathrm{a}}$ & $83,44 \pm 39,65^{a}$ & $60,67 \pm 28,82$ \\
\hline Effet période : & & GMQ (g) & \\
\hline Période 1 (P1) (92 ovins) & & $46,65 \pm 34,53^{\mathrm{a}}$ & \\
\hline Période 2 (P2) (92 ovins) & & $75,54 \pm 39,11^{b}$ & \\
\hline Effet genre & & GMQ (g) & \\
\hline Animaux des hommes (48 ovins) & & $75,39 \pm 32,23^{\mathrm{a}}$ & \\
\hline Animaux de femmes ( 42 ovins) & & $78,47 \pm 36,16^{\mathrm{a}}$ & \\
\hline
\end{tabular}

Classe : < 12 mois d'âge : classe $1 ; 12-18$ mois d'âge : classe $2 ;>18$ mois d'âge : classe 3 ; Période 1 (30 j) : temps entre la première et la troisième pesée sous la conduite paysanne (témoin); Période $2(60 \mathrm{j})$ : temps entre le début d'application du rationnement amélioré et la fin de l'essai ;Période $3(90 \mathrm{j})$ : Temps total mis pour les suivis des ateliers (temps mis pour le suivi des pratiques paysannes et du rationnement amélioré); Les moyennes figurant sur la même colonne et portant des lettres différentes sont significativement différentes au seuil de $5 \%$ et les chiffres inscrits dans les parenthèses indiquent les effectifs des animaux dans les lots.

\section{DISCUSSION}

Les résultats de l'étude montrent que dans la commune de Korsimoro, l'activité d'embouche est pratiquée aussi bien par les hommes que par les femmes et l'alimentation des animaux est basée essentiellement sur l'utilisation des ressources locales. La qualité de la plupart de ces ressources reste cependant faible et pourrait être liée en partie aux conditions de leur conservation. En effet, dans ces élevages comme dans la majorité des élevages traditionnels burkinabé, le stockage des fourrages se fait quasiment sur des hangars à ciel ouvert (Lodoun, 2012). Ce qui participe à dégrader davantage leur valeur nutritionnelle dans le temps.

Les valeurs obtenues dans l'étude en termes de quantité de rations paysannes distribuées ( 8 à $11 \mathrm{~kg} \mathrm{MS} / 100 \mathrm{~kg}$ de poids vif) sont nettement supérieures aux indications en offre de Rivière (1991) pour les ovins d'embouche qui varient de 3 à $6 \mathrm{~kg}$ MS pour $100 \mathrm{~kg}$ de poids vif. Les emboucheurs de Korsimoro distribuent par conséquent plus d'aliments qu'il en faut à leurs animaux. Dans leur logique et en rapport avec les observations de certains auteurs, cela ne constitue pas un gaspillage du fait qu'il permet aux animaux d'effectuer une consommation sélective qui améliore la qualité de l'ingéré en vue d'exprimer une croissance satisfaisante (Savadogo et al., 2000). Cette consommation sélective des aliments grossiers pourrait également expliquer le léger avantage en ingestion alimentaire pour les animaux du lot 1 comparativement aux animaux du lot 2 durant la phase du rationnement amélioré. En effet, le fait que les animaux du lot 1 n'aient pas bénéficié de la complémentation d'appoint au Bloc multinutritionnel pour mieux valoriser les aliments pauvres de leur ration, pourrait avoir incité ces derniers à les ingérer davantage en triant leurs parties les plus appétibles de manière à améliorer leur bol alimentaire. 
Tableau 4 : Compte d'exploitation d'opération d'embouche avec 2 têtes d'ovins.

\begin{tabular}{lcc}
\hline Libellés & Coûts/unité & Montant (F CFA) \\
\cline { 1 - 2 } A. COUTS DE PRODUCTION & & \\
1. Coûts variables & & \\
1.1. Frais sanitaires & 67,5 & 135 \\
1.2. Abreuvement & 66 & 132 \\
1.3. Alimentation & 8580 & 17160 \\
1.4. Prestations diverses (suivi véto, transport, etc.) & 91 & 182 \\
1.5. Achat des animaux à emboucher & 23335 & 46670 \\
Total coûts variables & & 64144 \\
\hline 2. Coûts fixes & & \\
2.1. Amortissement Matériel/équipement & 580 & 1160 \\
2.2. Amortissement Infrastructures & 1004,5 & 2009 \\
2.3. Main d'œuvre & 825 & 1650 \\
2.4. Frais financiers & - & - \\
Total coûts fixes & & 4819 \\
Coûts totaux & & 69098 \\
\hline B. PRODUITS DE L'EMBOUCHE & & \\
1. Vente animaux sur pieds/viande & 43434 & 86868 \\
2. Valeurs du fumier et autres & 288 & 576 \\
Total des produits & & 87444 \\
Marge brute globale (valeur des produits-valeur des charges & & 22589 \\
variables) (MB) & & \\
Marge brute (MB)/Animal & & 11295 \\
Marge nette globale (Total des produits-Coûts totaux) & & 18346 \\
Marge nette (MN)/animal & & 9173 \\
Ratio Avantage/coût (Total des produits/ Coûts totaux) & & 1,27 \\
\hline
\end{tabular}

Les valeurs d'ingestion volontaire enregistrées dans la présente étude restent globalement plus élevées que celles rapportées par Nignan et al. (2000) et celles relevées par Kiema et al. (2008a). Les chiffres obtenus par les premiers auteurs ont varié de 70 à $91 \mathrm{~g}$ $\mathrm{MS} / \mathrm{kg} \mathrm{PM}$ et ceux rapportés par les seconds ont varié entre 58 et $66 \mathrm{~g} \mathrm{MS} / \mathrm{kg}$ PM. Les niveaux élevés de l'ingestion des animaux de l'étude pourraient en partie s'expliquer par le fait que ces derniers étaient en majorité très jeunes (plus 66\% d'entre eux étaient âgés de moins de 12 mois). Les auteurs Zoundi. (2005) et Gnanda et al. (2008) ont fait observer que l'embouche avec les jeunes animaux doit tenir compte des besoins supplémentaires des croissances de ces derniers qui se traduiraient fréquemment pour une ingestion plus importantes d'aliments.

Les résultats en termes d'IC pour les deux modalités de rationnement expérimentées illustrent l'efficacité zooéconomique de la complémentation d'appoint $\mathrm{du}$ bloc multinutrionnel (BMN) dans les élevages à ressources alimentaires pauvres. Cependant, on note globalement que les valeurs indiciaires obtenues, restent élevées par rapport aux valeurs de celles attendues qui doivent être inférieures ou égales à 10 (Kondombo et Nianogo, 2001).

Les gains pondéraux totaux mesurés au cours de la présente étude sont moins performants que ceux rapportés par Kiema et al. (2008b) sur un essai conduit au Sahel 
burkinabé valorisant Cassia obtusifolia dans l'embouche de mouton Peul et qui ont varié de $6,4 \pm 2,4 \mathrm{~kg}$ à $8,0 \pm 4,6 \mathrm{~kg}$ en 76 jours d'alimentation.

Les GMQ obtenus de l'étude sous l'application du rationnement amélioré (72 à $79 \mathrm{~g} / \mathrm{j}$ ) sont meilleurs par rapport à ceux rapportés par Nignan et al. (2000) sur des béliers de même race et qui ont varié de 54 à $67 \mathrm{~g}$. Ils sont également plus intéressants que les résultats obtenus par Kondombo et Nianogo (2001).

Plusieurs auteurs (Ouédraogo et Gnanda, 2000) ; Gnanda et al., 2008) ont déjà observé la covariabilité entre les GMQ des animaux d'embouche et leur âge, et indirectement leurs poids initiaux. Les jeunes animaux qui sont toujours en phase de croissance, expriment habituellement les meilleurs GMQ, surtout lorsqu'ils sont soumis à une alimentation à haute valeur nutritive (Zoundi, 2005). Cependant, il reste le fait que d'autres sources (INERA, 2003) font observer que l'embouche avec les jeunes animaux rallonge toujours le temps des opérations et fait élever la facture de l'alimentation.

Deux raisons probables peuvent être avancées pour expliquer les résultats pondéraux légèrement plus performants des animaux des femmes de la présente étude. La première tient $\mathrm{du}$ fait que les animaux embouchés par ces dernières étaient globalement plus jeunes que ceux embouchés par les hommes. La seconde est que dans la réalité, sur le terrain, les femmes prennent plus soin de leurs animaux que les hommes. Les animaux des femmes sont toujours collés à elles et sont bien souvent considérés autant que les enfants de la famille (Boly et al., 2001).

Les résultats obtenus au cours de la présente étude en termes de marges bénéficiaires nettes par animal (9 173 F CFA) sont nettement plus intéressants que ceux obtenus par Nantoumé et al. (2009) sur des moutons de race Maure. La marge nette par tête rapportée par ces auteurs a varié de 3545 à $5850 \mathrm{~F} \mathrm{CFA}$ par animal et en fonction des lots. Un essai d'engraissement de béliers de race Djallonké conduit dans la région des plateaux au Togo, a permis de dégager des marges nettes encore moins intéressantes de 3 170 à 4222 F CFA par animal embouché (Pitala et al., 2012).

Au cours de leurs travaux, (Gnanda et al., 2005) ont noté que la rentabilité des ateliers d'embouche paysanne qu'ils ont étudiée a été améliorée de plus $250 \%$ grâce à l'application d'une ration de vulgarisation.

Le ratio avantage/coût de 1,27 obtenu dans cette étude est supérieur à ceux de 1,01 rapportés par Gnanda et al. (2008) sur les animaux d'embouche traditionnelle des éleveurs du Sahel burkinabé et de 1,08 par Nianogo et al. (1997) sur les animaux d'embouche vendus à Pouytenga, dans le Plateau Central du Burkina Faso.

\section{Conclusion}

Les résultats de l'étude donnent la preuve évidente de l'amélioration significativement possible des performances zootechniques des animaux embouchés sous gestion paysanne par un transfert participatif de ration éprouvée de recherche. Ils confirment également le rôle déterminant de complémentation d'appoint de $\mathrm{BMN}$ pour l'optimisation de l'utilisation des aliments locaux dans l'alimentation des animaux, mis en évidence à travers les performances pondérales enregistrées et les indices de consommation (IC) des rations appliquées. Cependant, il ressort des résultats globaux de l'étude que l'efficacité du rationnement testé aurait pu être plus plausible si le temps de mesure avait atteint au moins 90 jours, durée généralement recommandée pour les opérations d'embouche intensive. Cela est d'autant plus vraisemblable étant donné le fait que la majorité des animaux utilisés étaient très jeunes et avaient besoin d'un temps d'embouche plus long pour une réponse plus optimale au rationnement. L'association d'industriels à de telles opérations de 
recherche constitue une innovation qui permet d'assurer la durabilité de l'approvisionnement des producteurs en rations alimentaires finies pour leur besoin d'embouche.

En termes de rentabilité économique des ateliers d'embouche étudiés, les résultats de l'étude suggèrent la nécessité d'améliorer le dispositif organisationnel des emboucheurs afin de permettre à ces derniers de tirer meilleur profit des marchés locaux à bétail qui constituent les premières opportunités d'écoulement des animaux des petits exploitants. Le caractère marchandant et très aléatoire du marché à bétail (manque de tarification du prix du kg de poids vif) fait que les emboucheurs de petits effectifs de moutons (1 à 2 têtes) sont plus exposés au risque de pertes sèches. Dans cette perspective, le renforcement de la concertation des acteurs au sein de la plateforme d'innovation (PI) bétail-viande initiée par l'équipe de recherche dans cette commune dans le cadre du projet, reste l'option la plus plausible pour booster l'activité d'embouche ovine dans la localité.

Aussi, la disponibilité des ressources alimentaires locales globalement satisfaisante dans la zone d'étude, représente un atout très intéressant pour développer des stratégies de leur mobilisation et des techniques plus appropriées de leur valorisation dans la production de viande ovine.

\section{REMERCIEMENTS}

L'équipe de recherche traduit sa reconnaissance au Conseil Ouest et Centre Africain pour la Recherche et le Développement agricole (CORAF/WECARD) pour l'opportunité de financement qu'il lui a permis de mener cette recherche, grâce à ses appels à projets compétitifs. Elle tient à remercier le Gouvernement Australien à travers son ancienne agence AusAID actuellement DFAT (The australian Agency for International Development/ Department of Foreign And Trading) pour avoir soutenu le financement des activités de cette étude. Aux chercheurs de CSIRO (Commonwealth Scientific and Industrial Research Organisation) avec lesquels elle a collaboré dans le cadre de cette recherche, elle leur exprime toute sa gratitude.

\section{REFERENCES}

Boly H, Ilboudo JB, Oouédraogo M, Berti F, Lebally P, Leroy P. 2001. L'élevage du "mouton de case" : aspects techniques, socio-économiques et perspectives d'amélioration au Yatenga (Burkina Faso). Biotechnol. Agron. Soc. Environ., 5(4) : 201-208.

Gnanda BI, Nianogo JA, Zoundi SJ, Somda J, Koanda S. 2005. Performances techniques et économiques de l'embouche ovine en exploitation traditionnelle de la région sahélienne au Burkina Faso. CAMES Série A, 3: 49-56.

Gnanda BI, Samandoulgou Y, Tamboura HH, Sanou S, Kagoné H. 2008. Impact d'une démarche participative de transfert de techniques améliorées de rationnement sur les performances pondérales et financières de l'embouche ovine paysanne au Sahel burkinabé. Communication présentée à la $8^{\text {ème }}$ édition du Forum national de la recherche scientifique et des innovations technologiques (FRSIT), tenue du 29 novembre au 6 décembre 2008 à Ouagadougou, au Burkina Faso sous le thème : " Impact de la recherche scientifique, des inventions et des innovations sur les sociétés africaines", p. 353-364.

INERA (Institut de l'environnement et de recherches agricoles). 2003. Rapport d'activités du projet d'amélioration des performances de production laitière bovine et d'embouche ovine dans la zone de couverture de l'Union des groupements villageois d'Aribinda. Développement participatif de technologies (DPT). Institut de 
l'environnement et de recherches agricoles (INERA), $16 \mathrm{p}$.

Kiema A, Nianogo AJ, Ouedraogo T, Somda J. 2008a. Use of local feed resources in the farmers ram fatting scheme: technical and economical performance. Cahiers Agricultures, 17: 24-27.

Kiema A, Nianogo AJ, Somda J, Ouédraogo T. 2008b. Valorisation de Cassia obtusifolia L. dans l'alimentation des ovins d'embouche en région sahélienne du Burkina Faso. Tropicultura, 26(2): 98103.

Kondombo S, Nianogo JA. 2001. Performances d'ovins Djallonké alimentés à base de résidus de récoltes au Burkina Faso. Revue Agronomie Africaine, 13(2) : 59-66.

Lodoun B. 2012. Analyse des performances des opérations d'embouche bovine dans les régions centrales du Burkina Faso. Mémoire d'ingénieur du développement rural, option élevage, Institut du Développement Rural (IDR), Université polytechnique de Bobo-Dioulasso, Burkina Faso, 68 p.

MAHRH (Ministère de l'Agriculture, de l'Hydraulique et des Ressources Halieutiques). 2007. Rapport d'analyse des résultats préliminaires de la première phase du recensement général de l'agriculture, 2006-2010. Rapport d'étude, 260 p.

Nantoume H, Diarra CHT, Traore D. 2009. Performance and economic profit of upgrading poor roughages with cottonseed meal in fattening Maure sheep in Mali. Livestock Research for Rural Development, 21 (12). http://www.lrrd. org/lrrd21/12/nant21207.htm.

Nianogo AJ, Ouédraogo $\mathrm{O}$, Deuson R, Gnoumou B, Nassa S, Kaboré D. 1997. Étude des systèmes d'embouche commerciale dans la région de Pouytenga (Burkina Faso) In Actes de colloque de Développement des filières petits ruminants en régions chaudes : le rôle des organisations

d'éleveurs.

UCARDEC/Office de l'Elevage et des Pâturages, Djerba, 121-127.

Nignan M, Nianogo JA, sawadogo LL, Bougouma-Yaméogo V. 2000. Influence du taux de concentré sur l'ingestibilité, la croissance pondérale et le développement corporel du mouton Djallonké Mossi. Tropicultura, 18(4) : 171-176.

Ouédraogo T, Gnanda BI. 2000. Impact des interventions $\mathrm{du}$ PGRN-SY sur la productivité du bétail dans le Seno et le Yagha: situation de référence sur les activités d'embouche et de production laitière. Rapport définitif, Département Productions Animales, Dori, Burkina Faso, $50 \mathrm{p}$.

Pitala W, Yaokorin F, Bonfoh B, Boly H, Gbeassor M. 2012. Evaluation de la réponse du mouton Djallonké à l'embouche herbagère à Kolokopé au Togo. Livestock Research for Rural Development, 24 (1): http://www.lrrd.org /lrrd24/1/cont2401.htm.

Rivière R. 1991. Manuel de l'Alimentation des Ruminants Domestiques en milieu Tropical. Collection Manuels et Précis d'Elevage : Paris, France, 529 p.

Savadogo M, Zemmelink G, Nianogo JA. 2000. Effect of selective consumption on volontary intake and digestibility of sorghum, cowpea and groundnut straws in Djallonké rams. Animal Feed Science and Technology, 84: 265-277.

SCADD (Stratégie de croissance accélérée et de développement durable) 2011-2015. 2007. Ouagadougou, Burkina Faso, 114 p.

Somda J. 2001. Performances zootechniques et rentabilité financière des ovins en embouche au Burkina Faso. Biotechnol. Agron. Soc. Environ., 5(2): 73-78.

Van Soest PJ. 1994. Nutritional Ecology of Ruminants (2nd Edn). Cornell University: Press; 476p.

Yacouba S, Hassoumi G, Hamani D. 2004. Analyse technique et économique de 
l'embouche bobine et ovine dans trois zones agro-écologique du Niger. Rapport final d'étude. Direction des Etudes et de la Programmation/Ministère des Ressources Animales du Niger et Service de Coopération et d'Action Culturelles de l'Ambassade de France, Niamey, Niger, 96.

Zoundi JS. 2005. Systèmes d'Alimentation des ruminants au sein des exploitations mixtes agriculture élevage du plateau central au Burkina-Faso. Thèse de doctorat d'Etat, Université de Ouagadougou, $179 \mathrm{p}$.
Zoundi JS, Nianogo AJ, Sawadogo LL. 1996. Utilisation de gousses de Piliostigma reticulatum (DC.) Hochst. et de feuilles de Cajanus cajan (L.) Millsp. en combinaison avec l'urée pour l'engraissement des moutons Djallonké type Mossi et du Sud au Burkina. Tropicultura, 14: 149-152.

Zoundi SJ, Sawadogo LL, Nianogo JA. 2003. Pratiques et stratégies paysannes en matière de complémentation des ruminants au sein des systèmes d'exploitation mixte agriculture-élevage du plateau central et du nord du Burkina Faso. Tropicultura, 21: 122-128. 